\title{
A Literature Review of Cuckoo Search Algorithm
}

\author{
Manar Abdulkareem Al-Abaji \\ Computer Sciences Dept. , College of Education for Pure Science , University of Mosul, Iraq
}

\begin{abstract}
Optimization techniques play key role in real world problems. In many situations where decisions are taken based on random search they are used. But choosing optimal Optimization algorithm is a major challenge to the user. This paper presents a review on Cuckoo Search Algorithm which can replace many traditionally used techniques. Cuckoo search uses Levi flight strategy based on Egg laying Radius in deriving the solution specific to problem. CS optimization algorithm increases the efficiency, accuracy, and convergence rate. Different categories of the cuckoo search and several applications of the cuckoo search are reviewed.
\end{abstract}

Keywords: Cuckoo Search Optimization, Applications , Levy Flight

DOI: $10.7176 / \mathrm{JEP} / 11-8-01$

Publication date:March $31^{\text {st }} 2020$

\section{Introduction}

Optimization is a process of modifying a system to make some features to work more efficiently or finding alternative performance under given constraints, as possible by maximizing desired parameters and minimizing the undesired parameters which are involved in the problem. Maximizing means trying to obtain good results without the expense. If the computer or any Android phone is optimized then it runs faster or to run with fewer memory requirements. Optimization can be classified in many ways. cuckoo search algorithm is one of the algorithms in solving optimization problems (Venkata Vijaya Geeta \& Ravi Kiran Varma 2016).

Since the first introduction of Cuckoo Search (CS) by Xin-She Yang and Suash Deb in 2009 , the literature of this algorithm has exploded. Cuckoo search, which drew its inspiration from the brooding parasitism of cuckoo species in Nature, were firstly proposed as a tool for numerical function optimization and continuous problems. Researchers tested this algorithm on some well-known benchmark functions and compared with PSO and GA, and it was found that cuckoo search achieved better results than the results by PSO and GA. Since then, the original developers of this algorithm and many researchers have also applied this algorithm to engineering optimization, where Cuckoo search also showed promising results. Nowadays cuckoo search has been applied in almost every area and domain of function optimization, engineering optimization, image processing, scheduling, planning, feature selection, forecasting, and real-world applications(I. Fister Jr. et. Al 2014) .

\section{Cuckoo Search Algorithm}

Cuckoo search is a meta-heuristic algorithm inspired by the bird cuckoo, these are the "Brood parasites" birds. It never builds its own nest and lays their eggs in the nest of another host bird nest. Cuckoo is a best-known brood parasite. Some host birds can engage directly with the intruding cuckoo. If the host bird identifies the eggs that are not their egg then it will either throw that eggs away from its nest or simply rid its nest and build a new nest.

In a nest, each egg represents a solution and cuckoo egg represents a new and good solution. The obtained solution is a new solution based on the existing one and the modification of some characteristics. In the simplest form each nest has one egg of cuckoo in which each nest will have multiple eggs represents a set of solutions. CS is successfully used to solve scheduling problems and used to solve design optimization problems in structural engineering. In many applications like speech reorganization, job scheduling, global optimization.

Cuckoo search idealized such breeding behavior and can be applied to various optimization problems (XinShe Yang \& Deb 2010) .

1. Each cuckoo lays one egg at a time and dumps it in a randomly chosen nest.

2. The best nests with the high quality of eggs will carry to the next generations.

3. The number of available host nest is fixed and if a host bird identifies the cuckoo egg with the probability of pa $=0,1$ then the host bird can either throw them away or abandon them and build a new nest.

\section{Lévy Flights Mechanism}

Animals search for food in a random or quasi random manner in the nature. The foraging path of an animal is effectively a random walk because the next move is based on both the current location/state and the transition probability to the next location. The chosen directions probability modeled mathematically. Various studies had shown that the flight behavior of many animals and insects demonstrates the typical characteristics of Lévy flights. A Lévy flight is a random walk in which the step-lengths are calculated according to a heavy-tailed probability distribution. The distance from the origin of the random walk tends to a stable distribution after a large number of steps (S. Siva Prathyusha et.al 2018). 


$$
\mathrm{X}_{\mathrm{i}}(\mathrm{t}+1)=\mathrm{X}_{\mathrm{i}}(\mathrm{t})+\alpha \oplus \operatorname{Levy}(\lambda)
$$

Where $\alpha>0$ is the step size. In most of the cases assume that $\alpha$ is equal to one. The product $\oplus$ means entry-wise multiplication i.e. Exclusive OR operation Levy flight is a random walk with random step size following a levy distribution

$$
\text { levy } \sim \mathrm{u}=\mathrm{t}^{-\lambda}(1<\lambda \leq 3)
$$

\section{Algorithm 1 Original CS algorithm}

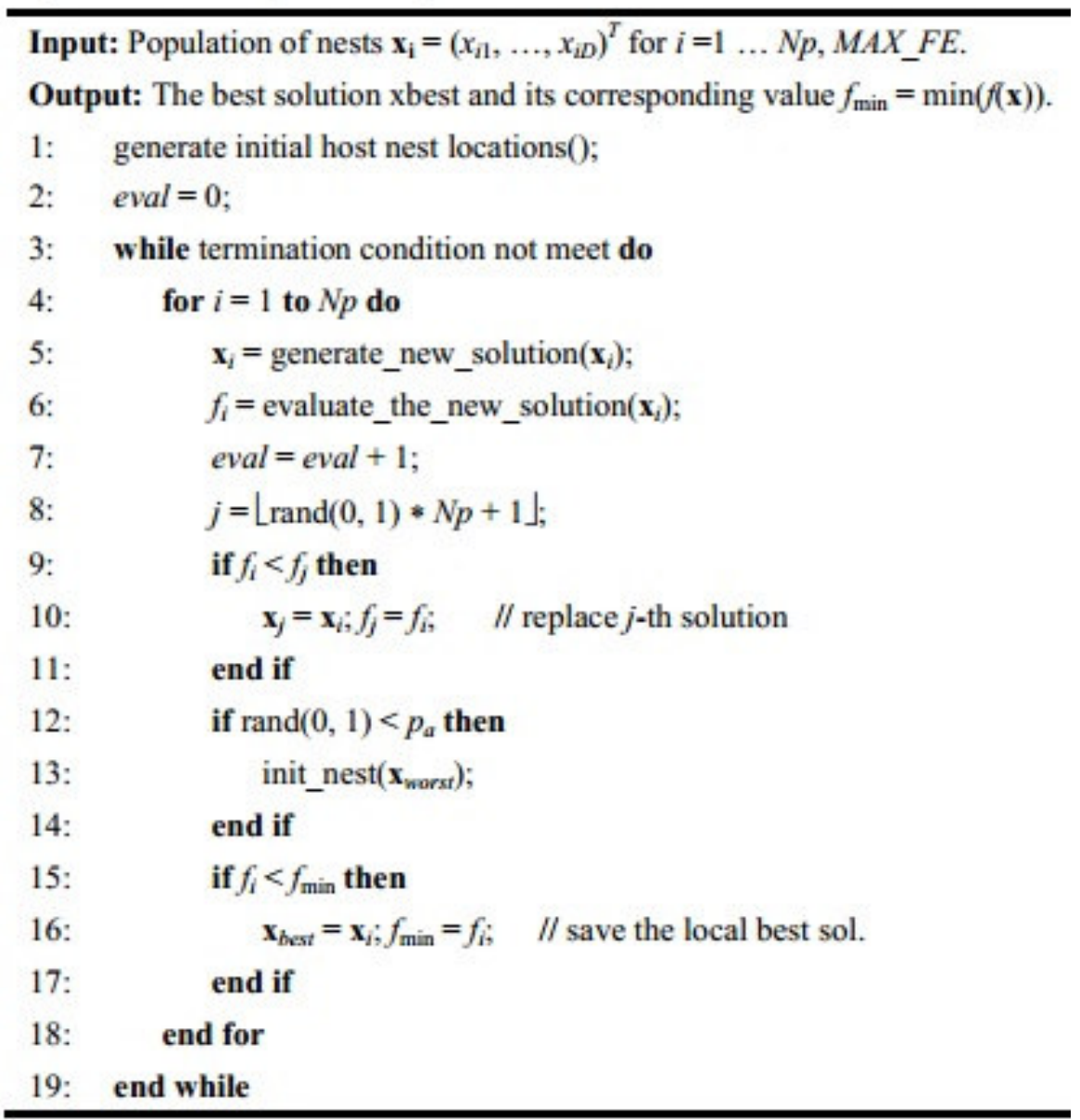

\section{Cuckoo Search: Variants and Hybrids}

\subsection{Variants}

The original cuckoo search was first tested using numerical function optimization benchmarks. Usually, this kind of problems represents a test bed for new developed algorithms. In line with this, standard benchmark function suites have been developed in order to make comparison between algorithms as fair as possible. For example, some original studies in this area are:

- Cuckoo search via L'evy flights .

- An efficient cuckoo search algorithm for numerical function optimization .

- Multimodal function optimization .

Cuckoo search can deal with multimodal problems naturally and efficiently. However, researchers have also attempted to improve its efficiency further so as to obtained better solutions or comparable results to those in the literature, and one such study that is worth mentioning is by Jamil and Zepernick .

Since the first appearance of cuckoo search in 2009, many variants of the cuckoo search algorithm have been developed by many researchers. The major variants are summarized in Table 1. 
Table 1 Variants CS

\begin{tabular}{lcc}
\hline Name & Author & Ref. \\
\hline Discrete binary CS & Gherboudj et al. & Gherboudj et al. (2012) \\
Discrete CS & Jati and Manurung & Jati et al. (2012) \\
Discrete CS for TSP & Ouaarab et al. & Ouaarab et al. (2013) \\
Binary CS & Rodrigues et al. & Rodrigues et al. (2013) \\
Neural-based CS & Khan and Sahai & Khan and Sahai (2013) \\
Quantum inspired CS & Layeb & Layeb (2011) \\
Emotional chaotic cuckoo & Lin et al. & Lin et al. (2012) \\
Cooperative coevolutionary & Zheng and Zhou & Zheng and Zhou (2013) \\
Cuckoo search-based LM & Nawi et al. & Nawi et al. (2013b) \\
Parallelised CS & Subotic et al. & Subotic et al. (2012) \\
Modified CS & Tuba et al. & Tuba et al. (2011) \\
Modified CS & Walton et al. & Walton et al. (2011) \\
Modified adaptive CS & Zhang et al. & Zhang et al. (2012) \\
A novel complex valued & Zhou and Zheng & Zhou and Zheng (2013) \\
CS-based on Gauss distribution & Zheng and Zhou & Zheng and Zhou (2012) \\
CS-based on Gaussian disturbance & Wang et al. & Wang et al. (2011b) \\
\hline
\end{tabular}

\subsection{Hybrid Algorithms}

For many continuous optimization problems, cuckoo search can find the desired solutions very efficiently. However, sometimes, some difficulty may arise, when the appropriate solutions could not be found for some other optimization problems. This is consistent with the so-called NoFree-Lunch theorem . To circumvent this theorem, hybridization has been applied to optimization algorithms for solving a given set of problems. In line with this, cuckoo search has been hybridized with other optimization algorithms, machine learning techniques, heuristics, etc. Hybridization can take place in almost every component of the cuckoo search. For example, initialization procedure, evaluation function, moving function and others have all been tried. Some of the hybrid variants are summarized in Table 2 .

Table 2 Hybrid CS

\begin{tabular}{lcc}
\hline Name & Author & Reference \\
\hline Hybrid CS/GA & Ghodrati and Lotfi & Ghodrati and Lotfi (2012a, 2012b) \\
Hybrid CS & Li and Yin & Li and Yin (2013) \\
\hline
\end{tabular}

\subsection{Multi-Objective Optimization}

Multi-objective optimization consists of optimizing the more than one objective, and these objectives may even be conflicting each another. Many real-world optimization problems require design solutions according to many criteria. While a single objective optimization algorithm searches for a single optimal solution, multi-objective optimization algorithms demand a set of many (potentially infinite), optimal solutions, namely the Pareto front in Robič and Filipič (2005), and Veldhuizen and Lamont (2000). Obviously, there are many issues and approaches for multi-objective optimization; however, two goals in multi-objective optimization are worth noting:

- to obtain solutions as close to the true Pareto front as possible

- to generate solutions as diversely as possible in the non-dominated front.

Various variants have been developed to extend the standard CS into multi-objective CS. Table 3 presents some main variants on multi-objective optimization using CS. 
Table 3 Multi-objective CS

\begin{tabular}{lcc}
\hline Name & Author & Reference \\
\hline $\begin{array}{l}\text { Multi-objective CS } \\
\text { Multi-objective scheduling problem }\end{array}$ & Yang and Deb & Yang and Deb (2013b) \\
$\begin{array}{l}\text { Multi-objective cuckoo search } \\
\text { algorithm for Jiles-Atherton vector } \\
\text { hysteresis parameters estimation }\end{array}$ & Coelho et al. & Chandrasekaran and Simon (2012) \\
$\begin{array}{l}\text { Pareto archived cuckoo search } \\
\begin{array}{l}\text { Hybrid multi-objective optimisation } \\
\text { using modified cuckoo search } \\
\text { algorithm in linear array synthesis }\end{array}\end{array}$ & Hanoun et al. & Coelho et al. (2013) \\
$\begin{array}{l}\text { Multi-objective cuckoo search for } \\
\text { water distribution systems }\end{array}$ & Wang et al. & Hanoun et al. (2012) \\
\hline
\end{tabular}

\section{Applications}

The applications of Cuckoo Search shown its promising efficiency. CS obtained better solutions than existing solutions in literature. Some of applications are:-

(Nima Jafari Navimipour et.al 2015) proposed a new evolutionary algorithm which named CSA to schedule the tasks in Cloud computing. CSA algorithm is based on the obligate brood parasitic behavior of some cuckoo species in combination with the Lévy flight behavior of some birds and fruit flies. The simulation results demonstrated that when the value of $\mathrm{Pa}$ is low, the speed and coverage of the algorithm become very high.

(Ehsan Valian et.al 2011) an improved cuckoo search algorithm enhancing the accuracy and convergence rate of the standard cuckoo search algorithm was proposed. Unlike the standard cuckoo search in which the parameters are kept constant, the ICS parameters are tuned. This results in increasing the efficiency of the algorithm. To evaluate the performance of the proposed algorithm, it is employed for training feed forward neural networks for two benchmark classification problems. Comparing the results provided by the proposed algorithm with those given by the CS demonstrates the effectiveness of the improved cuckoo search.

(Aminreza Noghrehabadi et.al 2011) applied hybrid Power series and Cuckoo Search via L'evy Flight Optimization algorithm (PS-CS) method to solve a system of nonlinear differential equations arising from the Distributed parameter model of a micro fixed-fixed switch subject to electrostatic force and fringing filed effect. A trial solution of the differential equation is defined as sum of two polynomial parts. The first part of it satisfies the boundary conditions and does not contain any adjustable parameter and the second part of it which is constructed so as not to affect the boundary conditions and involves adjustable parameters. PS-CS method is used here. This method provides more details about deflection shape of micro beams than lumped models. Micro fixed - fixed beams were computed using a combination of power series and heuristic Cuckoo Search optimization algorithm. This method is capable to obtain magnitude of bending moment and shear forces of micro fixed beam.PS-CS results are compared with the numerical results which show the PS-CS method using eight terms is in very good agreement with numerical results.

(Carlos Cobos et.ai 2014) introduced a new description-centric algorithm for the clustering of web results, called WDC-CSK, which is based on the cuckoo search meta-heuristic algorithm, k-means algorithm, Balanced Bayesian Information Criterion, split and merge methods on clusters, and frequent phrases approach for cluster labeling. The cuckoo search meta-heuristic provides a combined global and local search strategy in the solution space. Split and merge methods replace the original Lévy flights operation and try to improve existing solutions (nests), so they can be considered as local search methods. WDC-CSK includes an abandon operation that provides diversity and prevents the population nests from converging too quickly. Balanced Bayesian Information Criterion is used as a fitness function and allows defining the number of clusters automatically. WDC-CSK was tested with four data sets (DMOZ-50, AMBIENT, MORESQUE and ODP-239) over 447 queries. The algorithm was also compared against other established web document clustering algorithms, including Suffix Tree Clustering (STC), Lingo, and Bisecting k-means. The results show a considerable improvement upon the other algorithms as measured by recall, F-measure, fall-out, accuracy and SSLk.

(Ahmed T. Sadiq Al-Obaidi 2013) presented enhanced scatter search algorithm using CS algorithm. Testing is done on travelling salesman problem by original and improved Scatter Search. The Scatter Search (SS) is a deterministic strategy that has been applied successfully to some combinatorial and continuous optimization problems ,one of it is travelling salesman problem. The improvement in scatter Search with random exploration for search space of problem and more of diversity and intensification for promising solutions. The results are reported \& demonstrate that the improved Scatter Search algorithms produce better performance than original Scatter Search algorithm \&improvement in the value of average fitness is $23.2 \%$ comparing with original SS. It is 
found that The elapsed time for the improved SS is larger than the elapsed time for original SS in a reasonable value. The optimal solution of the improved SS is better than some algorithms but is far away from some others.

(Waleed Yamany et.al 2016) a system for attribute reduction was proposed using correlation-based filter model for attribute reduction. The cuckoo search (CS) optimization algorithm was utilized to search the attribute space with minimum correlation among selected attributes. Then, the initially selected solutions, guaranteed to have minor correlation, are candidates for further improvement towards the classification accuracy fitness function. The performance of the proposed system has been tested via implementing it using various data sets. Also, its performance have has been compared against other common attribute reduction algorithms. Experimental results showed that the proposed multi-objective CS system has outperformed the typical single-objective CS optimizer as well as outperforming both the particle swarm optimization (PSO) and genetic algorithm (GA) optimization algorithms

(S. Burnwal et.,al 2012) developed cuckoo search based approach for scheduling optimization of flexible manufacturing system by minimizing penalty cost due to delay in manufacturing and maximizing machine utilization time. To find optimum job, the proposed scheme has been applied with slight modification in its Levy Flight operator because of discrete nature of solution on standard FMS scheduling problem containing 43 jobs \& 16 machines which is taken from literature to demonstrate cuckoo search algorithm. Matlab is used to implement CS and results were compared with other soft computing based approaches such as Particle swarm optimization, Genetic algorithm. In flexible manufacturing system (FMS), scheduling is important decision making to decide effectiveness and capability of the system to increase operational efficiency. Cuckoo search based approach simplifies it.

(A.R. Yildiz 2012) used a newly developed Cuckoo search algorithm to solve manufacturing optimization problem. For that manufacturing optimization problem was solved to demonstrate effectiveness of Cuckoo Search \&results were compared with other algorithms such as immune algorithm, hybrid immune algorithm, hybrid particle swarm algorithm, genetic algorithm, ant colony algorithm. CS is performed effectively on the optimization of machining parameters of the milling operation problem finding better solutions compared to other approaches. $\mathrm{T}$ he CS is a generalized solution method so that it can be easily employed to consider the optimization models of milling regarding various objectives and constraints. The Cuckoo Search is very effective and robust approach for machining optimization problem.

(C. Gunavathi and K. Premalatha 2015) proposed Cuckoo Search (CS) optimization algorithm for feature selection in cancer classification using microarray gene expression data. Since the gene expression data has thousands of genes and a small number of samples, feature selection methods can be used for the selection of informative genes to improve the classification accuracy. Initially, the genes are ranked based on T-statistics, Signal-to-Noise Ratio (SNR) and F-statistics values. The CS is used to find the informative genes from the top-m ranked genes. The classification accuracy of k-Nearest Neighbour $(\mathrm{kNN})$ technique is used as the fitness function for CS. The proposed method is experimented and analysed with ten different cancer gene expression datasets. The results show that the CS gives 100\% average accuracy for DLBCL Harvard, Lung Michigan, Ovarian Cancer, AML-ALL and Lung Harvard2 datasets and it outperforms the existing techniques in DLBCL outcome and prostate datasets.

(G. Zheng et al 2012) proposed a three-step polynomial metamodel-assisted OP-AMP optimization flow ,address the issues of optimized OP-AMPs do not guarantee an optimal system performance.This paper presents such adesign flow for state-of-the art OP-AMP optimization. To estimate OP-AMP performance ultrafast and highly accurate polynomialmeta models are generated \& to facilitate fast time-domain simulations an OPAMP metamacromodel is constructed and is integrated into a Verilog-AMS module. Promised optimized results are produced by customizing cuckoo search algorithm. OP-AMP performance accurately predicted by OP-AMP characteristics with ultra-high speed. In the future result will be done on system-level optimization using the developed OP-AMP meta-macro model.

(Ahmed saadi Abdullah et.al 2019) a method was proposed to distinguish traffic signals based on curvelet transformations and cuckoo search algorithm Where the percentage of discrimination of traffic signs from one color to another different as the highest denominator to distinguish are for signs of traffic in red and then blue and then green and less is yellow where the proportion was about $96 \%$ of the red color, while the rest of the color was the proportion less. The proposed method was studied on different color systems where RGB, YCbCr, and $\mathrm{HSB}$ were used best results obtained when using the HSB color system. The efficiency of the algorithm was also measured in terms of image size.

(Aziz Ouaarab et.al 2013) told about an improved and discrete version of the Cuckoo Search algorithm to solve the famous Traveling Salesman Problem. The original cuckoo search algorithm is improved by introducing a new category of cuckoos so that it can solve combinatorial problems as well as continuous problems. Symmetric traveling salesman problem solved by using improved cuckoo search algorithm. By making study of interpretation of the terminology used in CS and in its inspiration source this adaptation is done. The goal of this Discrete CS is to give good ideas for designing new generations of more efficient meta-heuristic algorithms. Cuckoos have mimic 
nature so that very complex problems can efficiently solve by algorithms with apparent simplicity. They also want to develop new algorithms which will be more intelligent, more controllable \& less complex compared with other algorithms.

(M. Dhivya et.al 2011) developed Cuckoo based particle approach to achieve energy efficient Wireless Sensor Network and multimodal objective functions. Optimization of network is formulated by Cuckoo Based Particle Approach (CBPA) in this paper. In this case nodes are deployed randomly and organized as static clusters by Cuckoo Search (CS) algorithm \& After the cluster heads are selected, the information is collected, aggregated and forwarded to the base station by using generalized particle approach algorithm. Cuckoo search is applied for cluster head selection and formation of clusters among the Sensor nodes in this paper. After making comparison of proposed CBPA with standard LEACH protocol simulation results shows that CBPA produces comparable results mainly due to optimal search process in cluster formation and allocation of appropriate paths in transmission of sensed data. This developed algorithm reduces complexity in chain formation and prolongs the longevity of the Sensor Network. The final result obtained by doing number of simulation iterations.

(Lim Huai Tein 2010) used Cuckoo Search method to solve problems on Nurse scheduling. Nurse scheduling process is playing an important role in healthcare institutions around the world. So that there are few decades of studies that applying various techniques or algorithms to develop effective nurse schedule which from exploring optimization methods to search methods.

\section{Conclusion}

Cuckoo Search is being used as replacement of present used optimization algorithms. We focuses on the Cuckoo feeding behavior, Levy Flight mechanism and it's applications. Through its advent in 2009, this has been used in numerous applications such as scheduling, routing, choosing random variables, trace weights in neural networks, manufacturing, condition monitoring, health monitoring and so on. This can be compared with traditional optimization algorithm and is found to be best in the criteria.

\section{References}

Ahmed saadi Abdullah, Majida Ali Abed, Ahmed Naser Ismael, (2019), " Traffic signs recognition using cuckoo search algorithm and Curvelet transform with image processing methods", Journal of AL-Qadisiyah for computer science and mathematics, 11(2), $74-81$.

Ahmed T. Sadiq Al-Obaidi,(2013), "Improved Scatter Search Using Cuckoo Search", International Journal of Advanced Research in Artificial Intelligence 2, no. 2, 61-67

Aminreza Noghrehabadi, Mohammad Ghalambaz, Mehdi Ghalambaz, Amir Vosough,(2011), “A hybrid Power Series - Cuckoo Search Optimization Algorithm to Electrostatic Deflection of Micro Fixed-fixed Actuators", International Journal Of Multidisciplinary Sciences And Engineering 2, no. 4,22-26

A. R. Yildiz, (2012), "Cuckoo search algorithm for the selection of optimal machine parameters in milling operations", Int. J. Adv. Manuf. Technol .

Aziz Ouaarab, B. Ahiod, X.-S. Yang,(2013), "Discrete cuckoo search algorithm for the travelling salesman problem", Neural Computing and Applications,13(2).

Carlos Cobos, Henry Muñoz-Collazos, Richar Urbano-Muñoz, Martha Mendoza, Elizabeth León, Enrique Herrera-Viedma, (2014), " Clustering of web search results based on the cuckoo search algorithm and Balanced Bayesian Information Criterion", Information Sciences 281 (2014) , 248-264.

C. Gunavathi, K. Premalatha,(2015), " Cuckoo search optimisation for feature selection in cancer classification: a new approach", Int. J. Data Mining and Bioinformatics, 13( 3),248-265.

Chandrasekaran K. , Simon S.P. (2012) 'Multi-objective scheduling problem: hybrid approach using fuzzy assisted cuckoo search algorithm', Swarm and Evolutionary Computation, Vol. 5, pp.1-16.

Coelho L.S., Guerra F., Batistela N.J., Leite J.V., (2013),"Multiobjective cuckoo search algorithm based on duffings oscillator applied to Jiles-Atherton vector hysteresis parameters estimation', IEEE Transactions on Magnetics, Vol. 49, No. 5, p.1745.

Ehsan Valian, Shahram Mohanna, Saeed Tavakoli,(2011), " IMPROVED CUCKOO SEARCH ALGORITHM FOR FEEDFORWARD NEURAL NETWORK TRAINING", International Journal of Artificial Intelligence \& Applications (IJAIA), 2(3), 36-43.

Gherboudj A., Layeb A., Chikhi S. (2012),"Solving 0-1 knapsack problems by a discrete binary version of cuckoo search algorithm", International Journal of Bio-Inspired Computation, Vol. 4, No. 4, pp.229-236

Ghodrati, A. , Lotfi, S. (2012a) ,"A hybrid CS/GA algorithm for global optimization", in Proceedings of the International Conference on Soft Computing for Problem Solving (SocProS 2011), 20-22 December, 397404.

Ghodrati A. , Lotfi, S. (2012b) ,"A hybrid CS/PSO algorithm for global optimization", in Intelligent Information and Database Systems, Lecture Notes in Computer Science, Vol. 7198, 89-98.

G. Zheng, S. P. Mohanty, , E. Kougianos,(2012), "Metamodel-Assisted Fast and Accurate Optimization of an OP- 
AMP for Biomedical Applications”, in Proceedings of the 11th IEEE Computer Society Annual Symposium on VLSI (ISVLSI), 273-278.

Hanoun, S., Nahavandi S., Creighton D. , Kull H., (2012), "Solving a multiobjective job shop scheduling problem using Pareto archived cuckoo search", in 2012 IEEE 17th Conference on Emerging Technologies \& Factory Automation (ETFA), IEEE, 1-8.

I. Fister Jr., X. S. Yang, D. Fister, I. Fister,(2014), Cuckoo search: A brief literature review, in: Cuckoo Search and Firefly Algorithm: Theory and Applications, Studies in Computational Intelligence, vol. 516, 49-62 .

Jamil M. , Zepernick H-J. ,(2013) ,"Multimodal function optimisation with cuckoo search algorithm", International Journal of Bio-Inspired Computation, 5 (2), 73-83.

Jati G.K., Manurung H.M. , Suyanto, S. ,(2012) ,"Discrete cuckoo search for traveling salesman problem", in 7th International Conference on Computing and Convergence Technology (ICCCT2012), IEEE, 993-997.

Khan K., Saha A.,(2013),"Neural-based cuckoo search of employee health and safety (HS)", International Journal of Intelligent Systems and Applications (IJISA), 5 (2), 76-83.

Layeb, A. ,(2011),"A novel quantum inspired cuckoo search for knapsack problems", International Journal of Bio-Inspired Computation, 3(5), 297-305.

Lim Huai Tein , Razamin Ramli,(2010), "Recent Advancements of Nurse Scheduling Models and A Potential Path", Proceedings of the $6^{\text {th }}$ IMT-GT Conference on Mathematics, Statistics and its Applications (ICMSA2010), Universiti Tunku Abdul Rahman, Kuala Lumpur, Malaysia

Lin, J-H., Lee, H. et al., (2012) ,"Emotional chaotic cuckoo search for the reconstruction of chaotic dynamics", Latest Advances in Systems Science \& Computational Intelligence, WSEAS Press, Athens.

Li. X. , Yin. M., (2013) ,"A hybrid cuckoo search via Lévy flights for the permutation flow shop scheduling problem", International Journal of Production Research, (ahead-of-print), 51 (16), 1-23.

M. Dhivya, M. Sundarambal, L. N. Anand,(2011), "Energy Efficient Computation of Data Fusion in Wireless Sensor Networks Using Cuckoo Based Particle Approach (CBPA)", Int. J. of Communications, Network and System Sciences 4, no. 4, 249-255.

Nawi, N.M., Khan, A. , Rehman, M.Z. ,(2013b) ,"A new cuckoo search based Levenberg-Marquardt (CSLM) algorithm", in Computational Science and its Applications -ICCSA 2013, Springer, 438-451.

Nima Jafari Navimipour and Farnaz Sharifi Milani, (2015), " Task Scheduling in the Cloud Computing Based on the Cuckoo Search Algorithm", International Journal of Modeling and Optimization, 5 (1), 44-47.

Ong, P. and Zainuddin, Z., (2013), "An efficient cuckoo search algorithm for numerical function optimization", in AIP Conference Proceedings, Vol. 1522, p.1378

Ouaarab, A., Ahiod, B. and Yang, X-S. ,(2013),"Discrete cuckoo search algorithm for the travelling salesman problem", Neural Computing and Applications, pp.1-11, in press.

Rani, K., Malek, M.F.A., Neoh, S.C., Jamlos, F., Affendi, N.A.M., Mohamed, L., Saudin, N. and Rahim, H.A. ,(2012a),"Hybrid multiobjective optimization using modified cuckoo search algorithm in linear array synthesis", in Antennas and Propagation Conference (LAPC), Loughborough, IEEE, 1-4.

Rodrigues, D., Pereira, L., Almeida, T., Papa, J., Souza, A., Ramos, C. and Yang, X-S. ,(2013) ,"BCS: a binary cuckoo search algorithm for feature selection", in 2013 IEEE International Symposium on Circuits and Systems (ISCAS), IEEE, 465-468.

S. Burnwal, S. Deb,(2012), "Scheduling optimization of flexible manufacturing system using cuckoo search-based approach", Int. J. Adv Manuf Technol.

S. Siva Prathyusha, K. Sujatha, D. Harshitha, D. Sharmila, (2018), " Review On Cukoo Search Algorithm", International Journal of Management, Technology And Engineering, Volume 8, 2018.

Subotic, M., Tuba, M., Bacanin, N. and Simian, D. ,(2012),"Parallelized cuckoo search algorithm for unconstrained optimization", in Proceedings of the 5th WSEAS Congress on Applied Computing Conference, and Proceedings of the 1st International Conference on Biologically Inspired Computation, World Scientific and Engineering Academy and Society (WSEAS), 151-156.

Tuba, M., Subotic, M. , Stanarevic, N. ,(2011),"Modified cuckoo search algorithm for unconstrained optimization problems", in Proceedings of the 5th European conference on European Computing Conference, World Scientific and Engineering Academy and Society (WSEAS), 263-268.

Venkata Vijaya Geeta , Ravi Kiran Varma, (2016),"Cuckoo Search Optimization And Its Applications: A Review", International Journal of Advanced Research in Computer and Communication Engineering, 5(11).

Waleed Yamany, Nashwa El-Bendary, Aboul Ella Hassanien, Eid Emary, (2016), " Multi-Objective Cuckoo Search Optimization for Dimensionality Reduction", Procedia Computer Science 96 ( 2016 ), 207 - 215.

Walton, S., Hassan, O., Morgan, K. , Brown, M. ,2011) ,"Modified cuckoo search: a new gradient free optimisation algorithm", Chaos, Solitons \& Fractals, 44 (9), 710-718

Wang, F., He, X-s. , Wang, Y.,(2011b) ,"The cuckoo search algorithm based on Gaussian disturbance", Journal of Xi'an Polytechnic University, Vol. 4, p.27

Wang, Q., Liu, S., Wang, H., Savić, D. ,(2012d),"Multi-objective cuckoo search for the optimal design of water 
distribution systems", in Civil Engineering and Urban Planning 2012, ASCE, 402-405.

Xin-She Yang, Deb,(2010), "Engineering Optimization By Cuckoo Search" , J. Mathematical Modelling and Numerical Optimisation, 1(4).

Yang, X-S. , Deb. S. ,(2009) ,"Cuckoo search via Lévy flights", in World Congress on Nature \& Biologically Inspired Computing, 2009. NaBIC 2009, IEEE, 210-214

Yang, X-S. , Deb. S. ,(2013b) ,"Multiobjective cuckoo search for design optimization", Computers \& Operations Research, 40(6), 1616-1624

Zhang, Y., Wang, L. , Wu. Q. ,(2012) ,"Modified adaptive cuckoo search (MACS) algorithm and formal description for global optimization", International Journal of Computer Applications in Technology, 44( 2), 73-79

Zheng, H. , Zhou, Y. ,(2012),"A novel cuckoo search optimization algorithm based on gauss distribution", Journal of Computational Information Systems, Vol. 8, 4193-4200

Zheng H. , Zhou Y. ,(2013),"A cooperative coevolutionary cuckoo search algorithm for optimization problem", Journal of Applied Mathematics, Article ID 912056, 9pp, doi:10.1155/2013/912056.

Zhou Y., Zheng H., (2013),"A novel complex valued cuckoo search algorithm", The Scientific World Journal, Article ID 597803, 6pp, doi:10.1155/2013/597803. 